\title{
Parallel bias metadynamics and sketch-map dimensionality reduction as powerful tools to explore free energy landscapes of intrinsically disordered peptides
}

\author{
Olga Rogacheva \\ Laboratory of Biomolecular NMR, \\ SPbU, St. Petersburg, Russia \\ FSBRI "IEM", St. Petersburg, Russia \\ o.rogacheva@spbu.ru
}

\author{
Omar Valsson \\ MPIP, Mainz, Germany \\ valsson@mpip-mainz.mpg.de \\ Olga Shamova \\ FSBRI "IEM", St. Petersburg, Russia \\ shamova@iemspb.ru
}

\author{
Andrey Badanin \\ SPbU, St. Petersburg, Russia \\ a.badanin@spbu.ru
}

\begin{abstract}
A free energy landscape reconstruction is challenging even for a small intrinsically disordered peptide because conformational ensembles of intrinsically disordered peptides are heterogeneous and span numerous degrees of freedom. We combined two recently proposed modeling approaches, parallel bias metadynamics and sketch-map, to reconstruct and analyze the free energy landscape of the small peptide containing an RGD motif (HGRGDLGRLKK). This peptide originates from the transforming growth factor beta 3 proprotein and binds to the integrin aVb6 with high efficiency and selectivity. This makes it a promising agent for cancer and fibrosis drug development since integrin aVb6 is overexpressed in these pathological conditions. The simulations indicate that the peptide is mostly disordered; however, residues 4-7 exhibit a high percentage of the helical and turns structures. These residues are known to arrange in a helix when the peptide occupies the ligand-binding site of the integrin aVb6. So, the tendency of these residues to form helical structures in solution probably explains the high affinity of the peptide for the integrin. We will use these results in further simulations of chimeric peptides
\end{abstract} incorporating this peptide as a building block.

Keywords - intrinsically disordered peptides, parallel bias metadynamics, sketch-map, RGD-motif, free energy landscape

\section{Motivation and Aim}

A free energy landscape reconstruction is challenging even for a small intrinsically disordered peptide (IDP) because conformational ensembles of IDPs are heterogeneous and span numerous degrees of freedom. Defining stable states in this ensemble is also a non-trivial task. An RMSD-based clustering is not the best choice for IDPs since separation quality is achievable only at very small cutoff values. A dihedrals-based clustering also leads to a vast amount of different conformations that do not make sense, and we cannot interpret. Bearing in mind the challenges of IDPs modeling, we combined two recently proposed approaches, parallel bias metadynamics [1] and sketch-map [2], to reconstruct and analyze the free energy landscape of the small peptide containingan RGD motif (HGRGDLGRLKK). This peptide originates from the transforming growth factor beta 3 proprotein and binds to the integrin aV $\beta 6$ with high efficiency and selectivity [3]. This makes it a promising agent for cancer and fibrosis drug development since integrin $\mathrm{aV} \beta 6$ is overexpressed in these pathological conditions. The knowledge of the free energy landscape topology of this peptide will be helpful when introducing modifications into its sequence or using it as a building block for larger chimeric peptides. All these modifications may shift populations of the peptide conformations and result in a molecule that is incapable of receptor binding.

\section{Methods}

We used parallel bias metadynamics and the recently proposed ff99SB-disp force field [4], which is equally suitable for IDPs and folded proteins to explore the free energy landscape of the proposed peptide in solution. Biased potentials were applied along seven collective variables, including the similarity of the peptide to different regions of Ramachandran plot and order parameters of amide NH groups [5]. We furthermore performed long unbiased molecular dynamics simulations and compared the results with ones obtained in the parallel bias metadynamics simulations.

\section{Results}

On the limited time scales, parallel bias metadynamics efficiently samples a broader range of phase space than conventional molecular dynamics. Although these two methods converge in the low-lying minima well, only parallel biased metadynamics reproduce populations of high-energy states. Sketch-map is a dimensionality reduction algorithm that we used to build a $2 \mathrm{D}$ map of all peptide backbone dihedral angles. This allowed us to identify clusters of conformations with the elements of secondary structure and to calculate their populations. The simulations indicate that the peptide is mostly disordered with a tendency to form secondary structures of helical and beta-hairpin motifs. Perfect alpha helices and beta hairpins are poorly populated in the ensemble of conformations of this peptide as their population does not exceed 5\%. However, looking at individual residues, we observe that residues 4-7 exhibit a $20-50 \%$ population of helical and turn structures. These residues are known to arrange in a helix when the peptide occupies the ligand-binding site of the integrin $\mathrm{aV} \beta 6$. So, the tendency of these residues to form helical structures in solution probably explains the high affinity of the peptide for the integrin.

\section{REFERENCES}

[1] Pfaendtner J., Bonomi M. (2015) Efficient sampling of highdimensional free-energy landscapes with parallel bias metadynamics. Journal of chemical theory and computation. 11(11): 5062-5067.

[2] Ceriotti M. et al. (2011) Simplifying the representation of complex free-energy landscapes using sketch-map. PNAS, 108(32): 1302313028.

[3] Dong X.C., Hudson N.E., Lu C., Springer T.A. (2014) Structural determinants of integrin $\beta$-subunit specificity for latent TGF- $\beta$. Nature Structural \&Molecular Biology, 21(12): 1091-1096.

[4] Robustelli P., Piana S., Shaw D.E. (2018) Developing a molecular dynamics force field for both folded and disordered protein states. PNAS, 115 (21): E4758-E4766.

[5] Palazzesi F., Valsson O., Parrinello M. (2017) Conformational entropy as collective variable for proteins. 8(19): 4752-4756. 